\title{
Immunohistochemical evaluation of epithelial ovarian carcinomas identifies three different expression patterns of the MX35 antigen, NaPi2b
}

\author{
Kristina Levan ${ }^{1,5^{*}}$ (D), Matin Mehryar ${ }^{1}$, Constantina Mateoiu ${ }^{2}$, Per Albertsson ${ }^{3}$, Tom Bäck ${ }^{4}$ and Karin Sundfeldt ${ }^{1}$
}

\begin{abstract}
Background: To characterize the expression of the membrane transporter NaPi2b and antigen targeted by the MX35 antibody in ovarian tumor samples. The current interest to develop monoclonal antibody based therapy of ovarian cancer by targeting NaPi2b emphasizes the need for detailed knowledge and characterization of the expression pattern of this protein. For the majority of patients with ovarian carcinoma the risk of being diagnosed in late stages with extensive loco-regional spread disease is substantial, which stresses the need to develop improved therapeutic agents.

Methods: The gene and protein expression of SLC34A2/NaPi2b were analyzed in ovarian carcinoma tissues by QPCR $(n=73)$ and immunohistochemistry $(n=136)$. The expression levels and antigen localization were established and compared to the tumor characteristics and clinical data.

Results: Positive staining for the target protein, NaPi2b was detected for $93 \%$ of the malignant samples, and we identified three separate distribution patterns of the antigen within the tumors, based on the localization of NaPi2b. There were differences in the staining intensity as well as the distribution pattern when comparing the tumor grade and histology, the mucinous tumors presented a significantly lower expression of both the targeted protein and its related gene.

Conclusion: Our study identified differences regarding the level of the antigen expression between tumor grade and histology. We have identified differences in the antigen localization between borderline tumors, type 1 and type 2 tumors, and suggest that a pathological evaluation of NaPi2b in the tumors would be helpful in order to know which patients that would benefit from this targeted therapy.
\end{abstract}

Keywords: Ovarian cancer, NaPi2b expression, Monoclonal antibody, Radiotherapy

\section{Background}

MX35 is a monoclonal antibody targeting the sodiumdependent phosphate transport protein $2 \mathrm{~B}$ ( $\mathrm{NaPi} 2 \mathrm{~b}$ ) gene name SLC34A2. The normal expression is in epithelial cells like type II pneumocytes, brush border

\footnotetext{
*Correspondence: kristina.levan@gu.se

'Sahlgrenska Cancer Center, Department of Obstetrics and Gynecology, Institute of Clinical Sciences, University of Gothenburg, SE-405 30 Gothenburg, Sweden

${ }^{5}$ Sahlgrenska Cancer Center, Department of Obstetrics and Gynecology, Institute of Clinical Sciences, University of Gothenburg, S-413 45 Gothenburg, Sweden

Full list of author information is available at the end of the article
}

membrane of small intestine and in the mammary gland $[1,2]$. The protein is involved in actively transporting phosphate ions into the cell by a $\mathrm{Na}^{+}$co-transport [3-7]. Protein expression is further evident in female genital tract, endometrium, cervix and fallopian tube [8]. While normal ovary has been reported to lack expression of $\mathrm{NaPi}$ b the expression is high in epithelial ovarian cancer (EOC) NaPi2b is expressed in $80-100 \%$ of the tumors $[3,5,6,9,10]$. EOC is the most prevalent type of ovarian cancer $(90 \%)$, and consists of five pathological subtypes: serous, mucinous, clear cell, endometrioid and undifferentiated carcinoma [11]. Standard treatment 
includes optimal debulcing surgery followed by first-line chemotherapy in selected cases.

Current interest in targeting the $\mathrm{NaPi} 2 \mathrm{~b}$ protein in ovarian cancer by use of monoclonal antibodies either conjugated to alpha-emitting radionuclides [12-14], or as antibody drug conjugates [15] has highlighted the importance of evaluating the antigene expression in tumor samples. In the situation of using alpha emitting radionuclides i.e. targeted alpha therapy (TAT) for ovarian cancer, recently also explored for other antigenic targets than NaPi2b, a solution containing antibodies labeled with $\alpha$-particles emitting radionuclide is injected locally into the peritoneal cavity [16]. The short ranged $\alpha$ particles $(<0.1 \mathrm{~mm})$ used in TAT make them especially suitable to eradicate minimal residual disease, since a large portion of the radiation energy can be confined to the cancer cells only. At the same time, due to the short range a too large heterogeneity of the intratumoral distribution and/ or intensity of the antigen could impact the therapeutic outcome. This has been shown on epithelial ovarian cancer (EOC) biopsies where the tumor uptake (\%ID/g) radiolabeled MX35 could vary a factor of 20 in-between samples and that the activity uptake of MX35 correlated both with level and intensity of the MX35-antigen expression, as analyzed by autoradiography and immunohistochemistry [17]. Bioimaging of metastases in animal models of ovarian cancer has shown heterogenic distribution on small tumors of varying sizes $[18,19]$. Strategies to predict and counteract for the impact of heterogeneity are currently being investigated, including parameters like radiation crossfire and specific activity of the radiopharmaceutical [20].

Nevertheless, detailed information about the antigen expression pattern within the tumor mass, is crucial for small scale dose calculation and prediction of the biological outcome of the radiotherapy. Therefore, knowledge about the actual expression pattern of $\mathrm{NaPi} 2 \mathrm{~b}$ in different histologies, grades and stages of ovarian tumors (OT) is warranted.

In this report we analyzed the localization and expression pattern of the NaPi2b protein $(n=136)$ as well as its gene expression $(n=73)$ (SLC34A2) in fresh frozen ovarian borderline and malignant tumor samples. The results are described and correlated to clinical pathology. The number of samples included in previous expression pattern studies of $\mathrm{NaPi} 2 \mathrm{~b}$ in ovarian cancer range from $n=14-50[4,7,9,21]$, and our objective was to establish the antigen expression in a larger set of EOC samples.

\section{Methods}

\section{Tumor samples}

Ovarian tumor tissues were subjected to analysis by quantitative polymerase chain reaction (QPCR) $(n=73$, benign $n=5$, borderline $n=11$, malignant $n=57$ ) and immunohistochemistry (IHC) $(n=150$, malignant $n=108$, borderline $n=42$ ) (histology, stage and grade are described in Table 1.). The tumor samples were collected prospectively and consecutively from patients diagnosed from March 2001 to September 2010 with suspected cystic pelvic tumor as part of another study [22]. Ovarian biopsies from 14 women without ovarian cancer were used as control tissue. The local ethical committee at the University of Gothenburg approved the study, and each patient gave her informed, written consent. All case diagnoses were reviewed by a gynecological pathologist using established morphologic criteria according to World health organization (WHO) 2003 [23]. Fresh frozen biopsies from each tumor were divided into two samples one was used for RNA extraction and the other one was paraffin embedded and used in the tissue micro array (TMA). The staining intensity and pattern were evaluated according to histology and the dualistic model presented by Shih et al. [24] Type I included low-grade (G1) serous, low-grade (G1) endometrioid, all clear cell, and mucinous carcinomas. Type II included high-grade (G2-G3) serous, high-grade (G2G3) endometrioid, undifferentiated carcinoma, and malignant mixed mesodermal tumors [25].

\section{Quantitative polymerase chain reaction (qPCR)}

RNA extraction was performed using QIAGEN RNeasy plus Mini Kit (QIAGEN, Germany) according to the manufacturer's manual, and the RNA concentration was

Table 1 Malignant and borderline samples included in the analysis

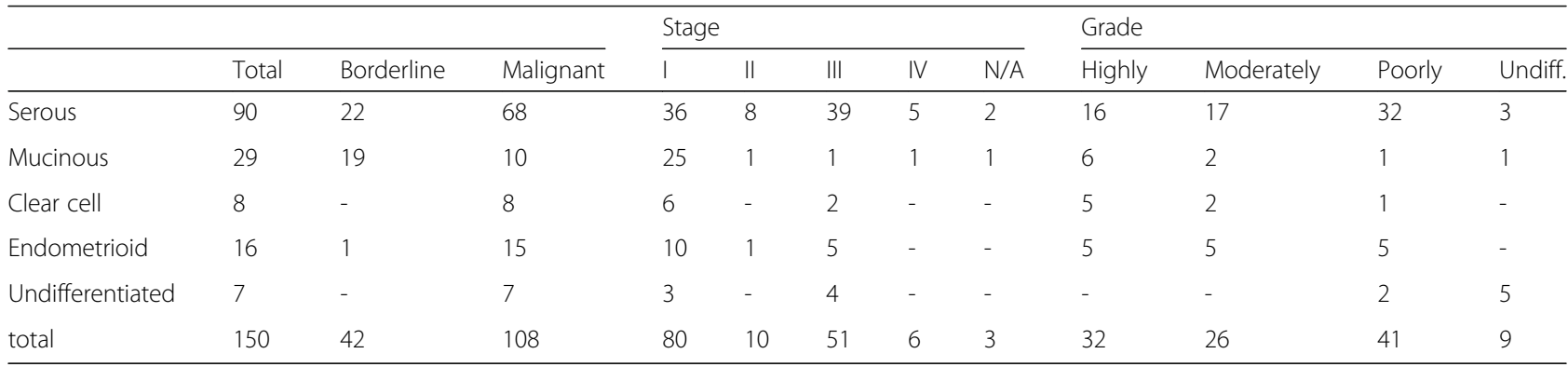

Presented based on histology, stage and grade respectively 
measured with the NanoDrop instrument (ND1000 software, Thermo Fisher Scientific, Wilmington, DE) (Table 1). The RT-PCR High-Capacity cDNA Reverse Transcription kit (Applied Biosystems, Foster City, CA) was used to produce cDNA from the RNA samples. TaqMan Universal PCR Master Mix (Applied Biosystems, Focter City, CA), probe and primers for SLC34A2 (Hs 00197519_m1) as the target gene and GUSB (Hs 99999908_m1) as the reference gene (Life Technologies Corporation, San Diego, CA) were used. A 7000 sequence detection system (Applied Biosystems, Foster City, CA) was used to determine the expression levels by QPCR of the target gene for all samples. Pooled normal ovarian tissue $(n=7)$ was used as control since it was previously reported to contain low levels of SLC34A2 [2, $5,21,26,27]$. The $\mathrm{Ct}$ values were used to calculate $\Delta \Delta \mathrm{Ct}$ and fold change (FC) for each tumor sample.

\section{MX35 antibody}

MX35 is a murine IgG1 monoclonal antibody specifically directed towards a membrane phosphate transporter protein (NaPi2b). The murine MX35 antibody was produced from a hybridoma line and was kindly provided by The Ludwig Institute for Cancer Research (New York, NY, USA). The hybridoma cells were cultured at the Department of Cell and Molecular Biology at the University of Gothenburg (Gothenburg, Sweden) and the antibody was purified from hybridoma supernatant by protein-A chromatography at the Department of Radiation Physics at the University of Gothenburg (Gothenburg, Sweden) [28].

\section{Immunohistochemistry (IHC)}

For the TMA, the whole biopsy was sectioned and stained with Hematoxylin (Histolab Products AB, Sweden). Three representative tumor areas were identified under the light microscope (Olympus BX45, Olympus Corporation, Tokyo, Japan), and three cores of $1,0 \mathrm{~mm}$-diameter were punched with a manual tissue microarrayer (Beecher MTA-1, Estigen,Tartu, Estonia) and re-embedded into a predefined position on a new, empty, paraffin block. The TMA block was heated at $45^{\circ} \mathrm{C}$ in $1 \mathrm{~h}$, sectioned, $4 \mu \mathrm{m}$, and mounted onto slides.

For IHC analysis, the TMA slides were immunostained by UltraVision Quanto Detection System HRP DAB kit (Thermo Fisher Scientific, Wilmington, DE) and incubated overnight with the MX35 antibody at a concentration of 1:1000. All slides were counterstained with hematoxylin and mounted with Pertex (Histolab Products $A B$, Sweden). All TMAs were scanned by a Leica SCN400 (Leica Microsystems, Milton Keynes, UK). SlidePath Gateway LAN software was used for the evaluation of the NaPi2b distribution.

\section{$\mathrm{NaPi}$ b expression}

Staining for NaPi2b were estimated for each tumor and the amount of positive cells were evaluated and given a value; no cells stained $=0,1 / 3=1,1 / 3>2 / 3=2,>2$ / $3=3$. The intensity was estimated for each tumor no staining $($ negative $)=0$, light yellow to yellow $($ weak $)=1$ + , light brown (moderate) $=2+$, and dark brown (strong) $=3+$. For the correlation analysis between QPCR and IHC we used a scoring system were we combined the intensity with the amount of cells stained in the tumor sample described by Tomic et al. [29]. The amount of cells stained (0-3) was used together with the intensity, to calculate a score that describes a combination of both the intensity and the amount of stained cell for each tumor. The product (amount of cells stained multiplied with intensity) ranging from zero to nine were grouped into four final scores as follows: score 0 , score 1 (low 1-3), score 2 (intermediate 4-6) score 3 (high >7) [29].

\section{Statistics}

The differences in expression of SLC34A2 between the groups previously described, were evaluated using unpaired two-sample Student's t-test $\left(\mathrm{IBM}^{\circ} \mathrm{SPSS}^{\circ}\right.$ Statistics) and were considered significant if $P<0.05$. In the analysis all samples were compared to the normal ovarian expression level, which was set to one. Correlation between the gene and protein expression was calculated using Pearson correlation. ANOVA test was used to analyze the variance of NaPi2b expression between the groups. Box plots of tumors grouped into stage, grade and histology were drawn to illustrate the ANOVA analysis results. Two researchers (MC and $\mathrm{KL}$ ) independently evaluated the IHC staining of the TMAs. In order to evaluate their inter-rater agreement Cohen's kappa coefficient was calculated.

\section{Results \\ SLC34A2 gene expression analysis}

To compare the expression levels of the SLC34A2 gene, coding for NaPi2b, among the different classifications of the ovarian tumors (OT), we subdivided the samples into groups based on histology, grade and stage (Table $1)$. The gene expression analysis of SLC34A2 displayed considerable variation in expression levels of this gene within the material, with values ranging from no expression up to a $\mathrm{FC}>1600($ mean $=237 ;$ median $=126)$ compared to the expression in normal ovaries. The mucinous OT demonstrated a significantly lower expression of SLC34A2 than both the serous and the clear cell OT ( $P=0.007$ and $P=0.002$ respectively) (Fig. 1a). We found no significant difference between mucinous and endometrioid OT $(P=0.062)$. Endometrioid OT had 

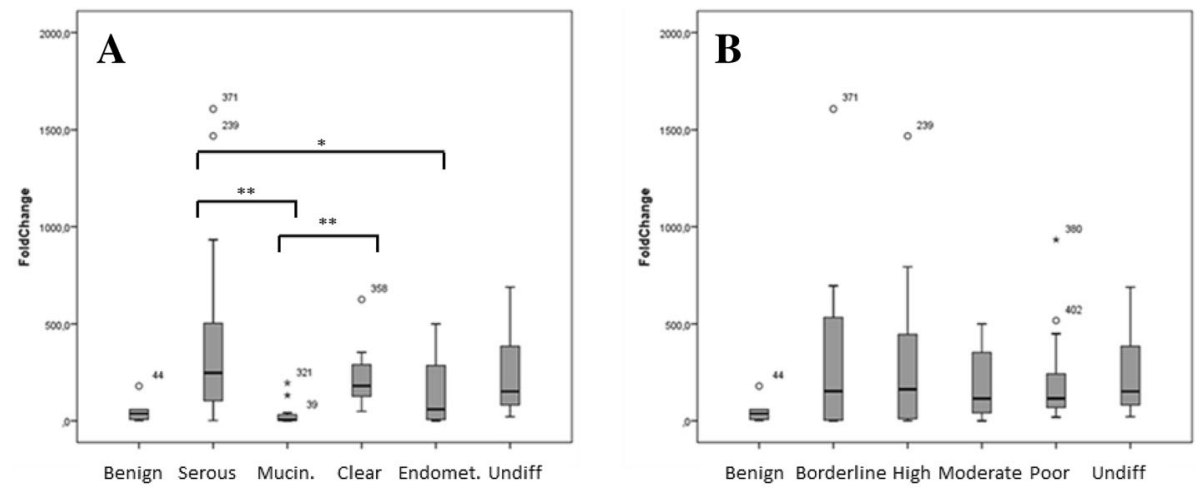

Benign Borderline High Moderate Poor Undiff
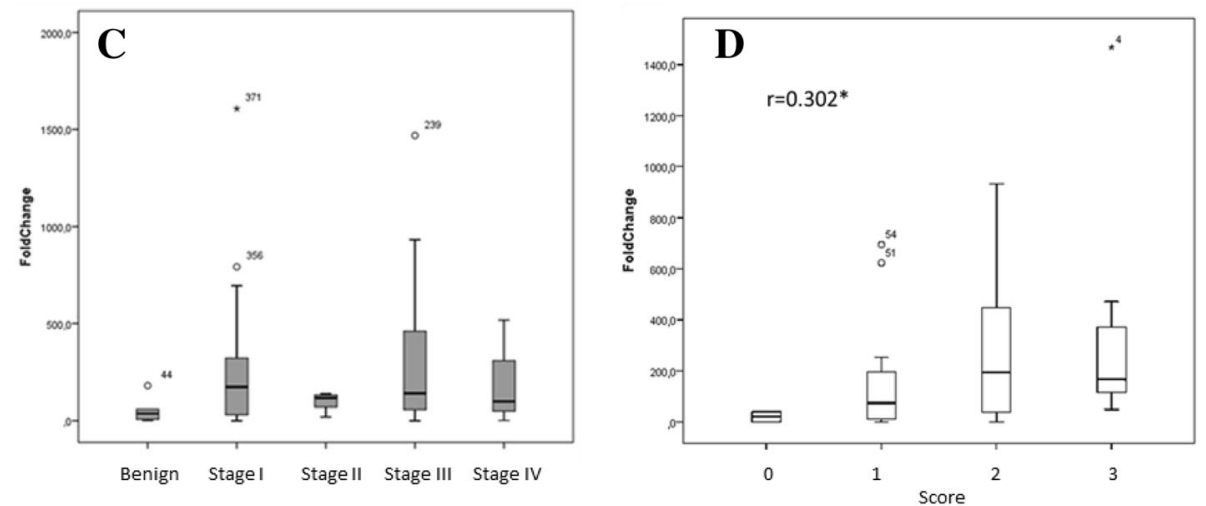

Fig. 1 Boxplots illustrating the level of SLC34A gene expression. a) level of expression in relation to the different histologies, with significant differences in expression between mucinous and both clear cell and serous ( ${ }^{* *} P<0.01$ ), and significant differences between endometrioid and serous tumors $\left({ }^{*} P<0.05\right.$, ); b) in relation to grade and in $\left.\mathbf{c}\right)$ to stage. $\left.\mathbf{d}\right)$ Boxplot illustrates the correlation between the staining of NaPi2b and the gene expression of SLC34A, Pearson correlation $r=0.302^{*}$

significantly lower expression levels than the serous OT $(P=0.038)$ (Fig. 1a).

\section{MX35 staining of $\mathrm{NaPi} 2 \mathrm{~b}$}

For evaluation of the $\mathrm{NaPi} 2 \mathrm{~b}$ expression in the tumors, six TMAs containing a total of 150 ovarian OT samples were stained with the MX35 antibody and scanned for analysis, 108 malignant and 42 borderline tumors (Table 1 ). Of the 150 OT 14 samples (9\%) were excluded from the analysis (ten malignant and four borderline) due to lack of tumor cells in the TMA. For the remaining 136 samples, quantification of the staining intensity and localization of NaPi2 was performed. For interrater reliability, Cohen's kappa coefficient was calculated for intensity $(\kappa=0.77)$ and for pattern $(\kappa=0.89)$, which established a robust IHC assessment. We found that 127 (93\%) out of the 136 samples were positively stained. A Pearson correlation analysis between the gene and protein expression in the tumor tissues was performed and a positive correlation was established $(r=0.302$, $P<0.05$ ) (Fig. 1d).

Among the 136 samples there were 41 tumors (30\%) stained at the highest level (3+), 48 tumors (36\%) as 2+, 38 tumors $(28 \%)$ as $1+$ and nine tumors (7\%) did not show any staining at all (Fig. 2a-b). Six of the negatively stained tumors were mucinous borderline tumors. The three malignant tumors with no staining were all type 1 , two were mucinous adenocarcinoma, one highly and one moderately differentiated, and one was highly differentiated serous adenocarcinoma. The borderline tumors had a higher number of cases with $3+$ staining compared to the malignant tumors, $47 \%$ and $29 \%$ respectively.

We subdivided the material according to histology, grade and stage. When comparing the histologies we were able to identify differences in the staining intensity between the groups (Fig. 2b). The serous tumors showed highest number of tumors with $3+$ staining between the histologies, and among the mucinous tumors only two out of 29 were considered to be $3+$ both of them were borderline tumors (Fig. 2c). The majority of the mucinous samples had negative or $1+$ staining in both the malignant (75\%) and in the borderline tumors (81\%). The low expression of the target protein $\mathrm{NaPi} 2 \mathrm{~b}$ in the mucinous tumors correlates well with the low gene expression of SLC34A2 in this group. The malignant tumors were grouped according to type (type $1: n=33$, type 2 : $n=65)[22,24]$, among the type 1 tumors $48 \%(n=16)$ had $1+$ or no staining, compared to only $25 \%$ with $1+$ 


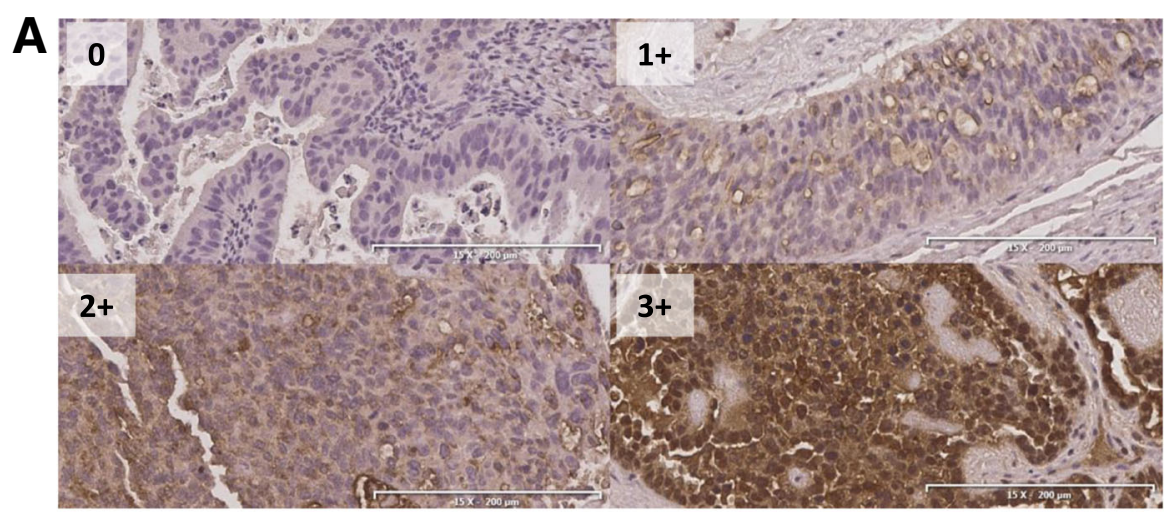

B
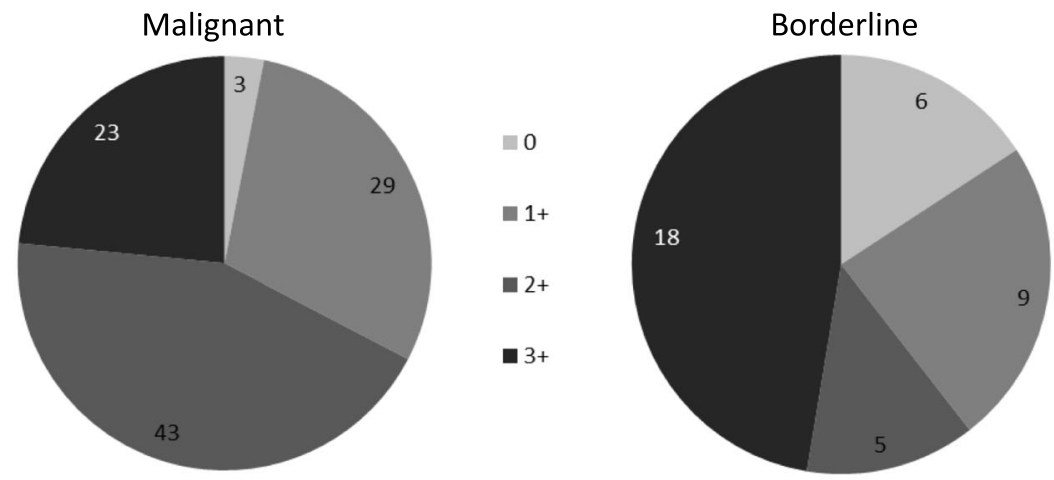

\section{C}

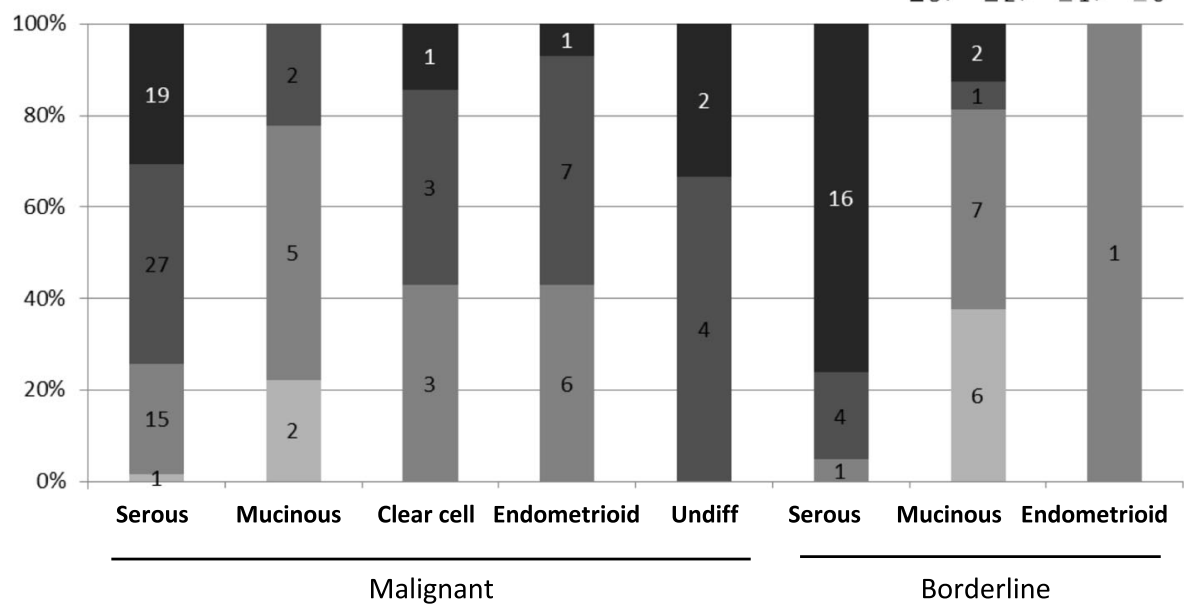

Fig. 2 Illustrative images of the staining intensities and the distribution of the different intensities among the samples. a) Representative images demonstrating the different staining intensities Upper left: no staining $=0$, serous (highly differentiated stage I), Upper right: $1=$ weak staining (endometrioid poorly differentiated, stage II). Lower left: 2 = moderate staining (endometrioid poorly differentiated stage III. Lower right: $3=$ strong staining, serous poorly differentiated stage III). b) Malignant and borderline tumor samples divided in scored staining intensity. c) Bars illustrating the samples divided into histology and how the staining intensities were distributed within their histological group

tumors in the more aggressive type 2. Further, there were $75 \%$ of the tumors that were considered as $2+$ or 3 + in the type 2 tumors (Fig. 3).

\section{Distribution pattern of $\mathrm{NaPi} 2 \mathrm{~b}$}

Because of the protein function and results from previous studies it was expected to find $\mathrm{NaPi} 2 \mathrm{~b}$ located to cell membranes $[3,4,6,7]$. When we evaluated the TMAs we identified differences in the staining pattern between the tumors (Fig. 4). We identified three different patterns for the distribution of NaPi2b (Fig. 4). In pattern A) NaPi2b was primarily located in the cellmembranes of cells close to the surface of the tumor. Even when the tumor consisted of several layers of epithelial cells 


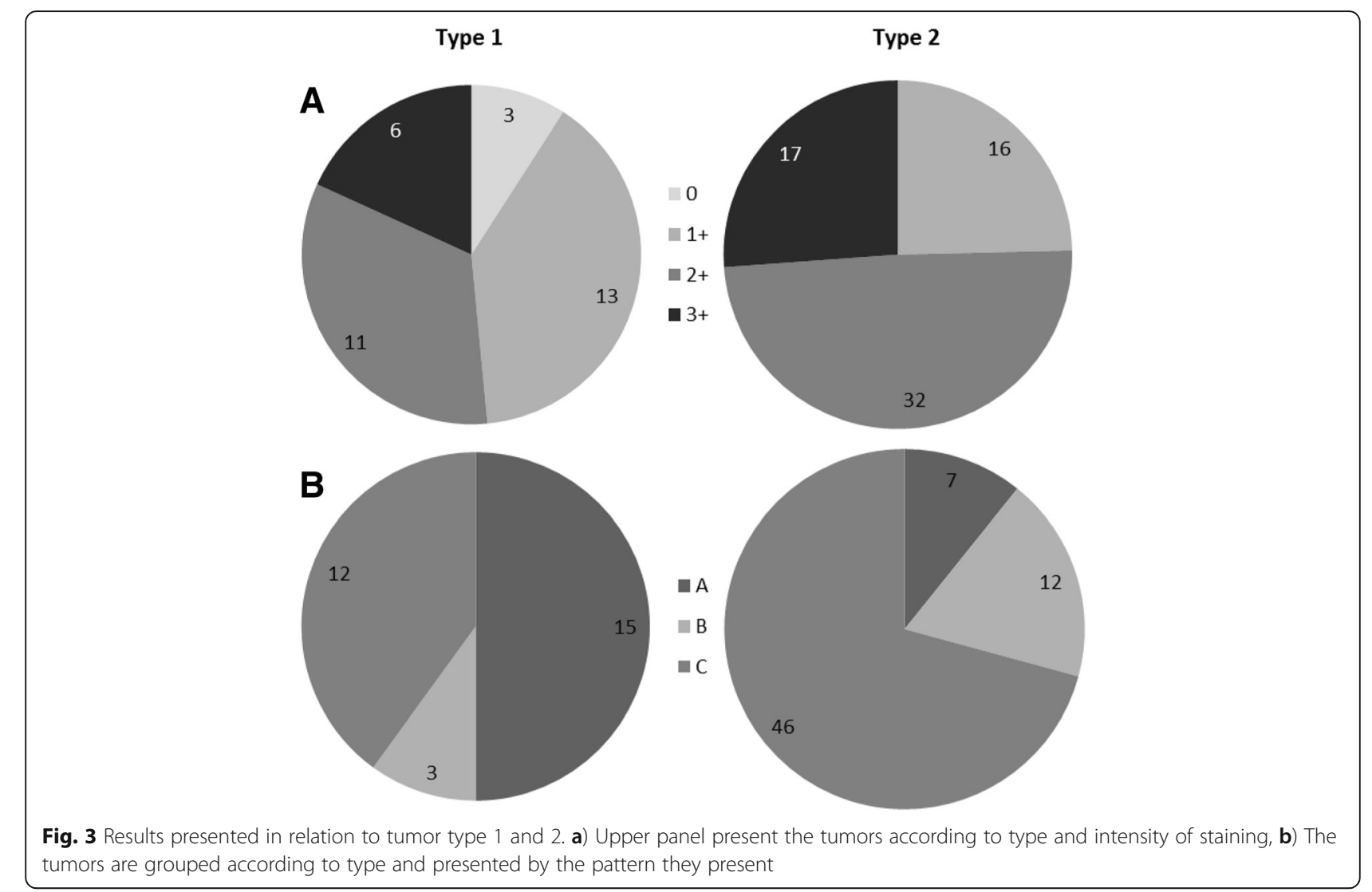

staining was only detected for epithelial cells that were located in the external layer of the tumor (Fig. 4a). In pattern B) NaPi2b was not limited to the cell membranes in the cells at the surface of the tumor but was found in all of the tumor cell membranes. Finally, in pattern $\mathrm{C}$ ) there was a mixed staining pattern with $\mathrm{NaPi} 2 \mathrm{~b}$ localized to both the cellmembranes and the cytoplasm of the same cell (Fig. 4c). Staining of normal ovarian tissue ( $n=4$ women) showed absence of MX35 in follicles, stroma and ovarian surface epithelium in $3 / 4$, and one had typical pattern A staining of ovarian surface epithelium only. Two of 3 women with endometriosis, originating from the uterus, had pattern A staining (data not shown). Of the 136 tumors, borderline and malignant, we were able to subdivide 126 samples into three groups according to the staining pattern (A, B and $\mathrm{C})$. The majority of borderline tumors $(n=29,90 \%)$ had pattern $\mathrm{A}$, and only three borderline tumors had both membrane and cytoplasmic, pattern $\mathrm{C}$ and none showed pattern $\mathrm{B}$ (Fig. 4d). Conversely, the majority of samples among the malignant tumors displayed pattern $C(n=57,61 \%)$ (Fig. 4d). Both pattern A and B were represented among these tumors, $23 \%$ and $16 \%$ respectively. With regard to histology, pattern $\mathrm{C}$ was most common in the serous (64\%), endometrioid (71\%) and the undifferentiated OT (67\%). Pattern A was the most common in mucinous
OT (67\%) (Fig. 4e). All three patterns were represented in the seven clear cell OC (A $n=2, \mathrm{~B} n=3, \mathrm{C} n=2$ ). None of the mucinous and endometrioid tumors displayed pattern B (Fig. 4e). In type 1 OT pattern A was present in $50 \%(n=13)$ of the cases, contrary to type 2 OT were the most frequent was pattern $\mathrm{C}$ which was detected in $71 \%(n=46)$.

\section{Discussion}

The main objective of this study was to characterize the expression of the NaPi2b in ovarian tumor samples. The development, evaluation and optimization of the targeted antibody treatment for this patient group call for a more detailed characterization of the cancer cell antigen expression. Our results from this study complement the present knowledge of $\mathrm{NaPi} 2 \mathrm{~b}$ expression in epithelial ovarian cancer and ovarian borderline tumors.

With $93 \%$ of the ovarian cancer tissue samples positively stained our data shows a higher frequency of $\mathrm{NaPi} 2 \mathrm{~b}$ expression compared to a study performed by Lopes dos Santos et al. where $80 \%$ of the ovarian cancers express the protein [10]. Among the samples positive for $\mathrm{NaPi} 2 \mathrm{~b}$ the samples were evenly distributed between the staining intensities. All of the type 2 tumors were positive for NaPi2b and three out of four tumors had moderate or strong staining. Strong staining was more 


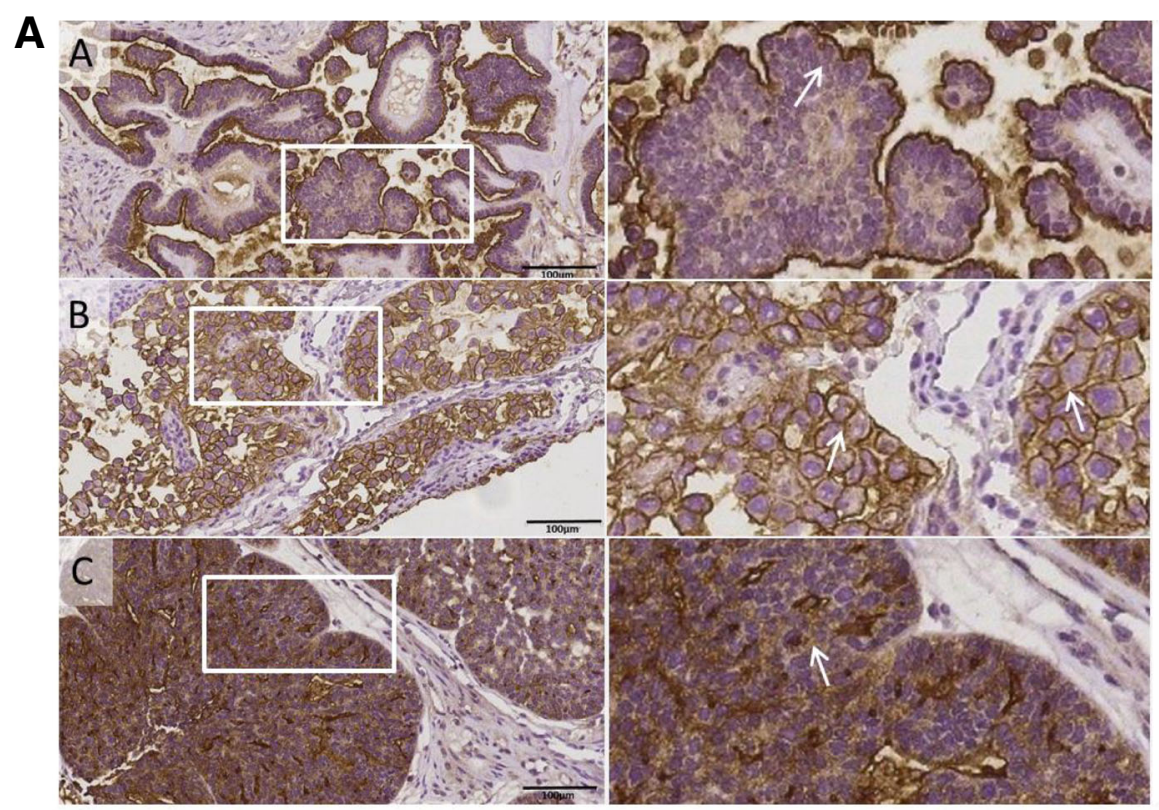

B
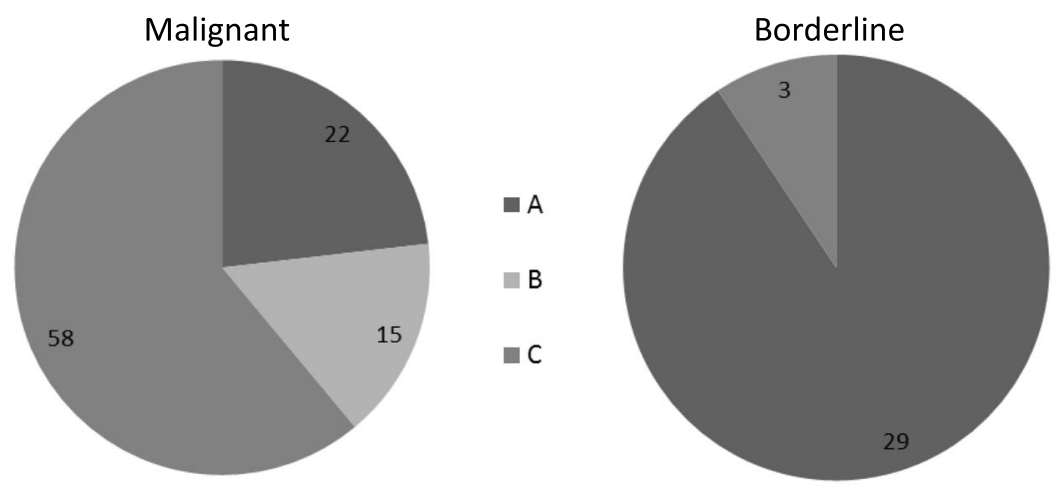

C

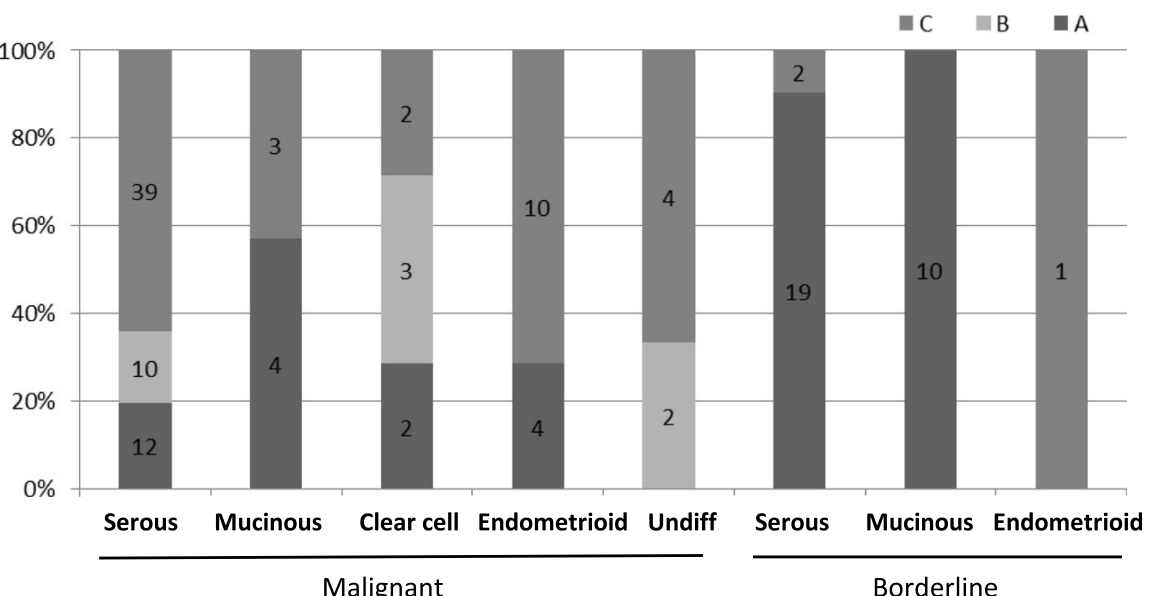

Fig. 4 Illustration presenting the three indicated staining patterns and their distribution among the samples. a) Illustration of the three characteristic MX35 staining patterns identified among the samples; Pattern $\mathbf{a}$, the target was located in the cell membranes of the cells close to the surface of the tumor (serous borderline, stage I). Pattern b, MX35 staining in all of the tumor cell membranes over the entire tumor (clear cell highly differentiated, stage I). Pattern c, includes the tumors with a mixed staining pattern including staining of membranes in addition to cytoplasmic staining spreading inside the as well (serous poorly differentiated stage I). The right panel shows images with higher magnification to give a more detailed view of the three patterns. b) The distribution of the tumor samples, malignant and borderline, between the three patterns. $\mathbf{c}$ ) The tumors divided based on pattern, presented according to their histology 
frequent among the serous borderline tumors, which is promising if targeted antibody based radiotherapy will be used in this group of patients. On the other hand, the majority of the mucinous tumors, both malignant and borderline, had low or negative staining compared to the serous tumors suggesting that theses tumors are not the ones that would benefit from this therapy. MX35 is designed to target $\mathrm{NaPi} 2 \mathrm{~b}$ expressed in the ovarian tumor cells and the antibody is suitable to carry a radionuclide that can deliver its energy to the target cells. It has been reported that other cancer types such as lung cancer, renal cancer and thyroid cancer also express the $\mathrm{NaPi} 2 \mathrm{~b}$ antigen, which suggests that this antibody may be useful in treatment of other cancer.

The MX35 staining patterns varied between the tumors, introducing novel information on how the antigen is distributed in the tissue. We classified the tumors according to three different staining patterns, and we believe that these differences in localization of the antigen are important factors governing the uptake and efficiency of a potential therapy targeting this protein. In the database, Human Protein Atlas, the pattern A, staining of the apical membrane, facing the surface of the tumor, was the dominating form represented in the samples shown for different types of tissue, including normal fallopian tubes, uterus and lung. For the normal tonsil there was an example with staining only in the cytoplasm, which would represent a forth pattern which is not represented in this study [30]. We found that normal ovaries were mostly without staining. If present, like in endometriosis lesion, pattern A was noticed, which is well in line with previous data $[2,5,21,26,27]$. In samples taken from cancer tumors presented at the human protein atlas database the most common pattern was $\mathrm{A}$, but there were a few samples that showed some cytoplasmic staining in addition to staining at the surface of the cells [30]. In contrast to the findings of Shyian et al., who describe staining of $\mathrm{NaPi} 2 \mathrm{~b}$ predominantly at the surface membrane of cancer cells in well differentiated serous and endometrioid ovarian cancer [21], we identified pattern $C$ (both membrane and cytoplasm) as the most common pattern for the malignant tumors, represented in $61 \%$ of the tumors. For borderline samples pattern A was presented in $90 \%$ of the cases and the remaining tumors showed pattern $C$. In concordance with Soares et al., we identified pattern B, staining in all the membrane of all layers of cancer cells, as the least common pattern with fifteen malignant tumors presenting pattern B [7].

There were differences in the expression levels of $\mathrm{NaPi}$ b between histologies i.e. in clear cell carcinoma the levels of MX35 staining was of higher intensity than for the mucinous tumors (Fig. 2), this was in agreement with previous study by Soares and colleagues [7]. The expression of SLC34A2 differed significantly among the histological groups with a less pronounced expression mainly in the mucinous tumors but also in the endometrioid tumors (Fig. 1). In contrast to previous studies we did not see any typical association between increased expression of SLC34A2 and differentiation grade of the tumors $[5,26]$. On the contrary we were able to identify distinct differences when the NaPi2b staining was examined in relation to type, rather than differentiation grade. Type 2 tumors had higher staining intensity and presented more tumors with pattern $\mathrm{C}$. High intensity staining in the cells could be a beneficial quality for the use of tailored immunotherapy strategies.

We detected low levels of the gene expressed in the benign samples, where the levels were within the same range as the mucinous malignant tumors, further emphasizes the importance of analyzing the expression of MX35 staining in biopsies from patients in order to ensure whether or not the patient could benefit from this type of therapy. In the majority of the samples the gene and protein expression correlated, but the inconsistencies between the gene and protein expression could be explained by the use of two separate tumor pieces, even though they were taken from the same tumor sample. In work with patient material it is important to acknowledge the heterogeneity of the tumors, both clinically and with respect to the tumor biology.

Ovarian cancer is characterized by unspecific symptoms and late diagnosis. At the time of diagnosis the majority of women have advanced stage disease with metastatic spread primarily in the abdominal cavity. It is therefore hypothesized that radio immunotherapy with the $\alpha$-particle emitter ${ }^{211}$ At bound to a MX35 antibody has the potential to be beneficial for such patients. This type of targeted therapy may be used after primary staging and debulking surgery, which includes at least the removal of all visible tumor mass, both adnexa, the uterus and the omentum. Preclinical studies with ovarian cancer have established the efficiency and toxicity of this treatment supporting this notion [31, 32].

The interest in using $\alpha$-particles in targeted therapy is increasing and TAT is being considered for many different cancers [14]. The TAT-regimen under current development, using MX35 with the $\alpha$-particle emitter ${ }^{211} \mathrm{At}$, is a consolidating loco-regional therapy aimed to treat peritoneal microscopic disease in patients relapsing after surgery and chemotherapy [12]. The translation to clinical trial was made after very promising results in a series of preclinical studies [31-33]. With future developments, TAT therapy may be complemented with a systemic regimen aimed to treat vascularized and/or extra-peritoneal tumors. For this purpose, the MX35 antibody could possibly be used non-radiolabeled as suggested by data from a recent study of Rebmab200, a 
humanized version of MX35 [10]. In a recent study the murine MX35, used in the present study, was compared with its humanized counterpart (Rebmab200) and the antigen binding properties and in vivo behavior were found to be very similar [28]. Further, with the implementation of a pre-targeted radioimmunotherapy, a systemic TAT might be a possibility for the future. For these targeted strategies the anti-tumor efficacy will depend on the antigen expression and its intra-tumoral distribution i. e the targeted antigen [10, 34]. We have previously shown that, due to the short range of $\alpha$ particles, the levels of absorbed radiation dose to the tumors, and other organs, could vary greatly depending on the distribution of radiolabeled antibody $[18,19]$.

There has been an increase in the use of antibodies within the field of targeted therapy $[14,35]$. The antigen $\mathrm{NaPi} 2 \mathrm{~b}$ is currently being explored as a target for antibody based immunotherapy in ovarian and pulmonary cancer $[10,12,36]$. It is of fundamental importance to know the antigen expression frequency as well as the cellular localization of the antigen before treatment, this will be especially important for the targeted radiotherapies involving short-ranged $\alpha$-particle irradiation, recently being explored for e.g. ovarian cancer [16, 37]. Our results suggest that there are differences regarding the level of the antigen expression between histologies and distinguish the mucinous tumors with a significantly lower expression of the antigen. Hence, a pathological evaluation of $\mathrm{NaPi} 2$ in the tumors that are surgically removed would give information on which patients that would benefit the most from a targeted therapy of this type. Furthermore, the presented data regarding the distribution of the $\mathrm{NaPi} 2 \mathrm{~b}$ antigen provide new knowledge for further development of antibody based therapy regimens of ovarian cancer.

\section{Conclusions}

Our study identified differences in the level of the antigen expression and in the antigen localization between borderline tumors, type 1 and type 2 tumors, and we therefore suggest that a pathological evaluation of $\mathrm{NaPi} 2 \mathrm{~b}$ expression in the tumors would be helpful in order to know which patients that would benefit from a therapy targeting this antigen.

\section{Abbreviations \\ EOC: Epithelial ovarian carcinoma; IHC: Immuno histo chemistr; OT: Ovarian tumors; QPCR: Quantitative polymerase chain reaction; TAT: Targeted alpha therapy; TMA: Tissue microarray; WHO: World health organization}

\section{Acknowledgements}

We wish to thank Birgitta Weijdegaard for skillful technical assistance in the laboratory and Teresia Kling for statistical consultation.

\section{Funding}

This research project was supported by the Swedish Cancer Foundation (PA, KS), local grants from WeCanCureCancer.com (KS), LUA-ALF- Agreement concerning research and education of doctors (PA, KS), the Assar Gabrielsson Foundation $(\mathrm{KL})$, the Hjalmar Svensson Foundation $(\mathrm{KL})$ and the King Gustav $\checkmark$ Jubilee Clinic Research Foundation (PA, TB). Neither of the funding bodies has been involved in the design of the study, the collection and analysis, or the interpretation of data in this manuscript.

\section{Availability of data and materials}

The datasets used and/or analysed during the current study available from the corresponding author on reasonable request.

\section{Authors' contributions}

$\mathrm{KL}$ designed the study and performed the statistical analysis as well as writing the manuscript. MM and $K L$ carried out the $I H C$ staining, the QPCR analysis and was involved in statistical analysis and the outlining of the manuscript. CM is the gynecological pathologist classifying the tumors as well as being one of the persons evaluating the $\mathrm{IHC}$ staining together with $\mathrm{KL}$. PA was involved in the design of the study and helped to draft the manuscript. TB participated in the design of the study, supplied the antibody and helped to draft the manuscript. KS was involved in the design of the study, the gathering and selection of the tumor material used in the study and helped to draft the manuscript. All authors read and approved the final manuscript.

\section{Competing interests}

The authors declare that they have no competing interests.

\section{Consent for publication}

Not appliable. This manuscript does not contain any individual persons data.

\section{Ethics approval and consent to participate}

The Regional Ethical Review Board in Gothenburg approved this research project in accordance with the Declaration of Helsinki. Each patient gave her informed written consent.

\section{Publisher's Note}

Springer Nature remains neutral with regard to jurisdictional claims in published maps and institutional affiliations.

\section{Author details}

${ }^{1}$ Sahlgrenska Cancer Center, Department of Obstetrics and Gynecology, Institute of Clinical Sciences, University of Gothenburg, SE-405 30 Gothenburg, Sweden. ${ }^{2}$ Department of Pathology and Cytology, Institute of Biomedicine, University of Gothenburg, SE-405 30 Gothenburg, Sweden. ${ }^{3}$ Department of Oncology, Institute of Clinical Sciences, University of Gothenburg, SE-405 30 Gothenburg, Sweden. ${ }^{4}$ Department of Radiation Physics, Institute of Clinical Sciences, University of Gothenburg, SE-405 30 Gothenburg, Sweden. ${ }^{5}$ Sahlgrenska Cancer Center, Department of Obstetrics and Gynecology, Institute of Clinical Sciences, University of Gothenburg, S-413 45 Gothenburg, Sweden.

Received: 22 August 2016 Accepted: 24 April 2017

Published online: 02 May 2017

\section{References}

1. Murer H, Forster I, Biber J. The sodium phosphate cotransporter family SLC34. Pflugers Arch. 2004:447(5):763-7.

2. $\mathrm{Xu} \mathrm{H}$, Bai L, Collins JF, Ghishan FK. Molecular cloning, functional characterization, tissue distribution, and chromosomal localization of a human, small intestinal sodium-phosphate (Na+-pi) transporter (SLC34A2). Genomics. 1999;62(2):281-4.

3. Yin BW, Kiyamova R, Chua R, Caballero OL, Gout I, Gryshkova V, Bhaskaran N, Souchelnytskyi S, Hellman U, Filonenko V, et al. Monoclonal antibody MX35 detects the membrane transporter NaPi2b (SLC34A2) in human carcinomas. Cancer Immun. 2008:8:3.

4. Rubin SC, Kostakoglu L, Divgi C, Federici MG, Finstad CL, Lloyd KO, Larson SM, Hoskins WJ. Biodistribution and intraoperative evaluation of radiolabeled monoclonal antibody MX35 in patients with epithelial ovarian cancer. Gynecol Oncol. 1993;51(1):61-6.

5. Gryshkova V, Goncharuk I, Gurtovyy V, Khozhayenko Y, Nespryadko S, Vorobjova L, Usenko V, Gout I, Filonenko V, Kiyamova R. The study of 
phosphate transporter NAPI2B expression in different histological types of epithelial ovarian cancer. Exp Oncol. 2009;31(1):37-42.

6. Kiyamova R, Shyian M, Lyzogubov W, Usenko VS, Gout T, Filonenko V. Immunohistochemical analysis of NaPi2b protein (MX35 antigen) expression and subcellular localization in human normal and cancer tissues. Exp Oncol. 2011;33(3):157-61.

7. Soares IC, Simoes K, de Souza JE, Okamoto OK, Wakamatsu A, Tuma M, Ritter G, Alves VA. In silico analysis and immunohistochemical characterization of $\mathrm{NaPi} 2 \mathrm{~b}$ protein expression in ovarian carcinoma with monoclonal antibody Mx35. Appl Immunohistochem Mol Morphol. 2012; 20(2):165-72.

8. Uhlen M, Fagerberg L, Hallstrom BM, Lindskog C, Oksvold P, Mardinoglu A, Sivertsson A, Kampf C, Sjostedt E, Asplund A, et al. Proteomics. Tissue-based map of the human proteome. Science. 2015;347(6220):1260419.

9. Mattes MJ, Look K, Furukawa K, Pierce VK, Old LJ, Lewis JL Jr, Lloyd KO Mouse monoclonal antibodies to human epithelial differentiation antigens expressed on the surface of ovarian carcinoma ascites cells. Cancer Res. 1987;47(24 Pt 1):6741-50.

10. Lopes dos Santos M, Yeda FP, Tsuruta LR, Horta BB, Pimenta AA Jr, Degaki TL, Soares IC, Tuma MC, Okamoto OK, Alves VA, et al. Rebmab200, a humanized monoclonal antibody targeting the sodium phosphate transporter NaPi2b displays strong immune mediated cytotoxicity against cancer: a novel reagent for targeted antibody therapy of cancer. PLoS One. 2013;8(7):e70332.

11. Prat J. Ovarian carcinomas: five distinct diseases with different origins, genetic alterations, and clinicopathological features. Virchows Arch. 2012; 460(3):237-49.

12. Andersson H, Cederkrantz E, Back T, Divgi C, Elgqvist J, Himmelman J, Horvath G, Jacobsson L, Jensen H, Lindegren S, et al. Intraperitoneal alphaparticle radioimmunotherapy of ovarian cancer patients: pharmacokinetics and dosimetry of (211)at-MX35 F(ab')2-a phase I study. J Nucl Med. 2009; 50(7):1153-60

13. Cederkrantz $E$, Andersson $H$, Bernhardt $P$, Back T, Hultborn R, Jacobsson $L$, Jensen $\mathrm{H}$, Lindegren S, Ljungberg M, Magnander T, et al. Absorbed doses and risk estimates of (211)at-MX35 F(ab')2 in Intraperitoneal therapy of ovarian cancer patients. Int J Radiat Oncol Biol Phys. 2015;93(3):569-76.

14. Elgqvist J, Frost S, Pouget JP, Albertsson P. The potential and hurdles of targeted alpha therapy - clinical trials and beyond. Front Oncol. 2014;3:324.

15. Lin K, Rubinfeld B, Zhang C, Firestein R, Harstad E, Roth L, Tsai SP, Schutten M, Xu K, Hristopoulos M, et al. Preclinical development of an anti-NaPi2b (SLC34A2) antibody-drug conjugate as a therapeutic for non-small cell lung and ovarian cancers. Clin Cancer Res. 2015;21 (22):5139-50.

16. Meredith RF, Torgue J, Azure MT, Shen S, Saddekni S, Banaga E, Carlise R, Bunch P, Yoder D, Alvarez R. Pharmacokinetics and imaging of $212 \mathrm{~Pb}$ TCMC-trastuzumab after intraperitoneal administration in ovarian cancer patients. Cancer Biother Radiopharm. 2014;29(1):12-7.

17. Finstad CL, Lloyd KO, Federici MG, Divgi C, Venkatraman E, Barakat RR, Finn RD, Larson SM, Hoskins WJ, Humm JL. Distribution of radiolabeled monoclonal antibody MX35 F(ab')2 in tissue samples by storage phosphor screen image analysis: evaluation of antibody localization to micrometastatic disease in epithelial ovarian cancer. Clin Cancer Res. 1997; 3(8):1433-42.

18. Chouin N, Lindegren S, Frost SH, Jensen H, Albertsson P, Hultborn R, Palm S, Jacobsson $L$, Back $T$. Ex vivo activity quantification in micrometastases at the cellular scale using the alpha-camera technique. J Nucl Med. 2013;54(8): 1347-53.

19. Chouin N, Lindegren $S$, Jensen $H$, Albertsson P, Back T. Quantification of activity by alpha-camera imaging and small-scale dosimetry within ovarian carcinoma micrometastases treated with targeted alpha therapy. Q J Nucl Med Mol Imaging. 2012;56(6):487-95.

20. Palm S, Back T, Haraldsson B, Jacobsson L, Lindegren S, Albertsson P. Biokinetic modeling and Dosimetry for optimizing Intraperitoneal Radioimmunotherapy of ovarian cancer Microtumors. J Nucl Med. 2016; 57(4):594-600

21. Shyian M, Gryshkova V, Kostianets O, Gorshkov V, Gogolev Y, Goncharuk I, Nespryadko S, Vorobjova L, Filonenko V, Kiyamova R. Quantitative analysis of SLC34A2 expression in different types of ovarian tumors. Exp Oncol. 2011; 33(2):94-8.

22. Kristjansdottir B, Levan K, Partheen K, Sundfeldt K. Diagnostic performance of the biomarkers HE4 and CA125 in type I and type II epithelial ovarian cancer. Gynecol Oncol. 2013;131(1):52-8.
23. Tavassoli FA, Devilee P. Pathology and Genetics of Tumours of the Breast and Female Genital Organs, vol. Chapter 2. Lyon: IARC Publications; 2003.

24. Shih le M, Kurman RJ. Ovarian tumorigenesis: a proposed model based on morphological and molecular genetic analysis. Am J Pathol. 2004;164(5): $1511-8$.

25. Kurman RJ, Shih le M. Molecular pathogenesis and extraovarian origin of epithelial ovarian cancer-shifting the paradigm. Hum Pathol. 2011;42(7): 918-31.

26. Rangel LB, Sherman-Baust CA, Wernyj RP, Schwartz DR, Cho KR, Morin PJ. Characterization of novel human ovarian cancer-specific transcripts (HOSTs) identified by serial analysis of gene expression. Oncogene. 2003;22(46): 7225-32.

27. Nishimura M, Naito $S$. Tissue-specific mRNA expression profiles of human solute carrier transporter superfamilies. Drug Metab Pharmacokinet. 2008; 23(1):22-44.

28. Lindegren S, Andrade LN, Back T, Machado CM, Horta BB, Buchpiguel C, Moro AM, Okamoto OK, Jacobsson L, Cederkrantz E, et al. Binding affinity, specificity and comparative Biodistribution of the parental Murine monoclonal antibody MX35 (anti-NaPi2b) and its humanized version Rebmab200. PLoS One. 2015;10(5):e0126298.

29. Tomic TT, Gustavsson H, Wang W, Jennbacken K, Welen K, Damber JE. Castration resistant prostate cancer is associated with increased blood vessel stabilization and elevated levels of VEGF and Ang-2. Prostate. 2012; 72(7):705-12.

30. Uhlén M, Fagerberg L, Hallström BM, Lindskog C, Oksvold P, Mardinoglu A, Sivertsson Å, Kampf C, Sjöstedt E, Asplund A, Olsson I, Edlund K, Lundberg E, Navani S, Szigyarto CA, Odeberg J, Djureinovic D, Takanen JO, Hober S, Alm T, Edqvist PH, Berling H, Tegel H, Mulder J, Rockberg J, Nilsson P, Schwenk JM, Hamsten M, von Feilitzen K, Forsberg M, Persson L, Johansson F, Zwahlen M, von Heijne G, Nielsen J, Pontén F. Tissue-based map of the human proteome. Science. 2015;347(6220):1260419.

31. Elgqvist J, Andersson H, Back T, Claesson I, Hultborn R, Jensen H, Johansson BR, Lindegren S, Olsson M, Palm S, et al. Alpha-radioimmunotherapy of intraperitoneally growing OVCAR-3 tumors of variable dimensions: outcome related to measured tumor size and mean absorbed dose. J Nucl Med. 2006;47(8):1342-50.

32. Elgqvist J, Andersson $H$, Back T, Hultborn $R$, Jensen $H$, Karlsson B, Lindegren S, Palm S, Warnhammar E, Jacobsson L. Therapeutic efficacy and tumor dose estimations in radioimmunotherapy of intraperitoneally growing OVCAR-3 cells in nude mice with (211)at-labeled monoclonal antibody MX35. J Nucl Med. 2005;46(11):1907-15.

33. Mulford DA, Scheinberg DA, Jurcic JG. The promise of targeted \{alpha\}particle therapy. J Nucl Med. 2005;46(Suppl 1):1995-204S.

34. Frost $\mathrm{SH}$, Back T, Chouin N, Hultborn R, Jacobsson L, Elgqvist J, Jensen $H$, Albertsson P, Lindegren S. Comparison of 211At-PRIT and 211At-RIT of ovarian microtumors in a nude mouse model. Cancer Biother Radiopharm. 2013;28(2):108-14

35. Mullard A. Maturing antibody-drug conjugate pipeline hits 30. Nat Rev Drug Discov. 2013;12(5):329-32.

36. Burris HA, Gordon MS, Gerber DE, Spigel DR, Mendelson DS, Schiller JH, Wang Y, Choi Y, Wood K, Maslyar DJ, et al. A phase I study of DNIB0600A, an antibody-drug conjugate (ADC) targeting NaPi2b, in patients (pts) with non-small cell lung cancer (NSCLC) or platinum-resistant ovarian cancer (OC). In: 2014 ASCO annual meeting. J Clin Oncol. 2014;32:5s.

37. Meredith R, Torgue J, Shen S, Fisher DR, Banaga E, Bunch P, Morgan D, Fan J, Straughn JM Jr. Dose escalation and Dosimetry of first-in-human alpha Radioimmunotherapy with 212Pb-TCMC-Trastuzumab. J Nucl Med. 2014 55(10):1636-642. 